\title{
MEDICO-A Simple IoT Integrated Medical Kiosk for the Rural People
}

\author{
Sunil Jacob ${ }^{1}$ and Varun G Menon ${ }^{2}$ \\ ${ }^{1}$ Center for Robotics, SCMS School of Engineering and Technology, India \\ ${ }^{2}$ Department of Computer Science and Engineering, SCMS School of Engineering and \\ Technology, India
}

\begin{abstract}
After conducting a detailed survey among the villagers of Pallissery and Karukutty, it was observed that in most of the villages the native people have to travel long distances for their basic health needs. Also most of the villagers lack knowledge regarding live health updates. At times, these problems have even resulted in death of many people including pregnant women and children. The objective of our research is to propose an integrated and easy to use Medical Kiosk that can be installed at various locations in rural areas. The Kiosk will provide an integrated environment for all medical related activities and would perform numerous functions like sending notifications regarding medical camps, mobile medical help, important dates for vaccinations, child care, insurance policies and provide other live medical updates to the villagers. It would also support the basic facilities for measurement of body parameters like height, weight, BMI, blood pressure, and heartbeat and also facilitate live consultation facilities with specialized doctors through video and voice chats.
\end{abstract}

Keywords: Healthcare; Internet of Things; IoT; Medical Assistance; Medical Kiosk; Rural people

\section{INTRODUCTION}

Post conducting the survey among the villagers of Pallissery and Karukutty [1,2], it was observed that, in most of the villages, the native people have to travel long distances for their basic health needs. It was also found out that most of the villagers lack knowledge regarding live health updates. This, at times, have even resulted in death of many people including pregnant women and children. In the following paragraphs we discuss on the some of the major observations made during our survey.

Rural verses Urban Divide: Urban centers have numerous private hospitals and clinics which provide quality healthcare. These centers have better doctors, access to preventive medicine, and quality clinics which are a result of better profitability for investors compared to the not-so-profitable rural areas. The rural population mostly relies on alternative medicine and government programs in rural health clinics. However, these have proven ineffective till date.

Need for Effective Payment Mechanisms: Most Indian patients pay for their hospital visits and doctors' appointments with straight up cash after care with no payment arrangements. Only less than 1 percentage of Indian village population are aware of health insurance policies 
Demand for Basic Primary healthcare and infrastructure: India faces a growing need to fix its basic health concerns in the areas of HIV, malaria, tuberculosis, and diarrhea. Additionally, children under five are born underweight and roughly $7 \%$ (compared to $0.8 \%$ in the US) of them die before their fifth birthday.

Lack of standardized drugs: Although, India proclaims itself as the third largest producer of drugs, it is a major concern that most of the medicines are of abnormal composition, out of date and unavailable to most of the villagers.

In this research work, we use the power of Internet of Things [3-8] and propose an efficient solution to this problem. A number of studies have been previously conducted on developing efficient healthcare applications using Internet of Things (IoT) [9-13]. We had gathered multiple inputs from the existing methods and literature and we developed a real time implementation of a successful Medical Kiosk that is explained in the next section.

\section{PROPOSED SOLUTION}

Contemplating the problem statements, our thought process ended up with the idea of a cubicle machine. The machine will provide an integrated environment offering various medical services, which includes:

- Notifications regarding medical camps, mobile medical help, important dates for vaccinations, child care, insurance policies etc., and other live medical updates

- Basic facilities for measurement of body parameters like height, weight, BMI, blood pressure, and heartbeat.

- Live consultation facilities with specialized doctors through video and voice chats.

- Instant check for availability of doctors in nearby hospitals.

- Options to find out the best hospitals for each disease.

- Information regarding subsidies from government.

- Instant emergency medical services like ambulance, and mobile hospitals if needed.

- Vending of tablets post consulting a doctor.

- User friendly environment, that supports almost all regional languages and can be accessed through fingerprint and RFID in ration cards.

The implementation of the above-mentioned solution has both hardware and software requirements. The idea is to create GUI and integrate all the required components into a single system. Connecting all systems together to user and vice versa is done by means of IoT technology. We have also studied on the latest fog computing technology [14-18] and in our future project we would try to bring the computing power much nearer to the devices.

Figure 1 shows the hardware setup of the proposed system 


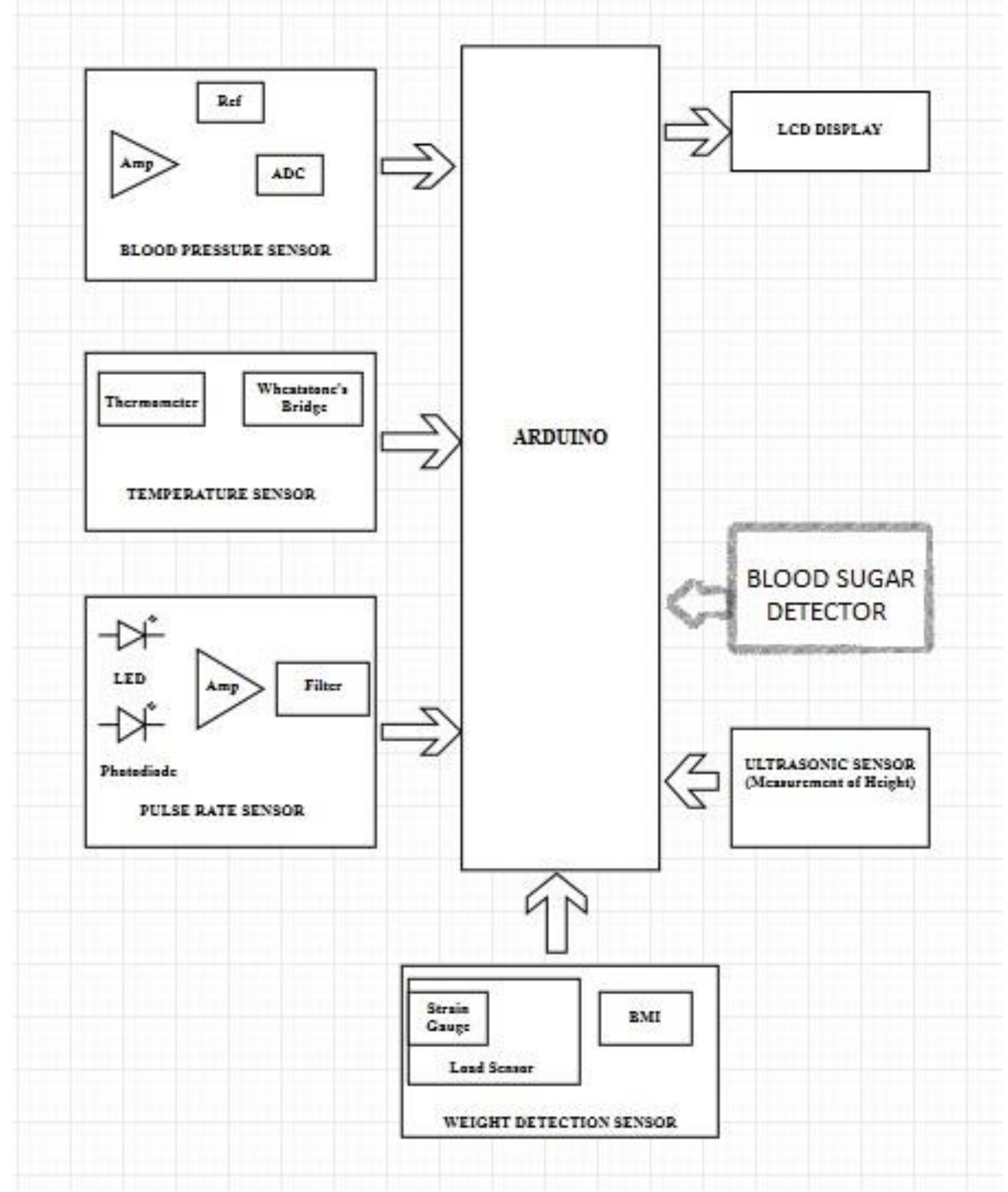

Fig 1: Hardware setup of the proposed system

It involves various components like pressure sensor for measuring blood pressure, temperature sensor for measuring body temperature, pulse-rate sensor for measuring heartbeat, weight detection sensor and load cells for measuring body mass, methyl sensor for measuring blood sugar levels \& ultrasonic sensor for measuring height. An Arduino controller is used and we make use of an LCD display for viewing. Figure 2 shows the circuit diagram of the proposed system. 


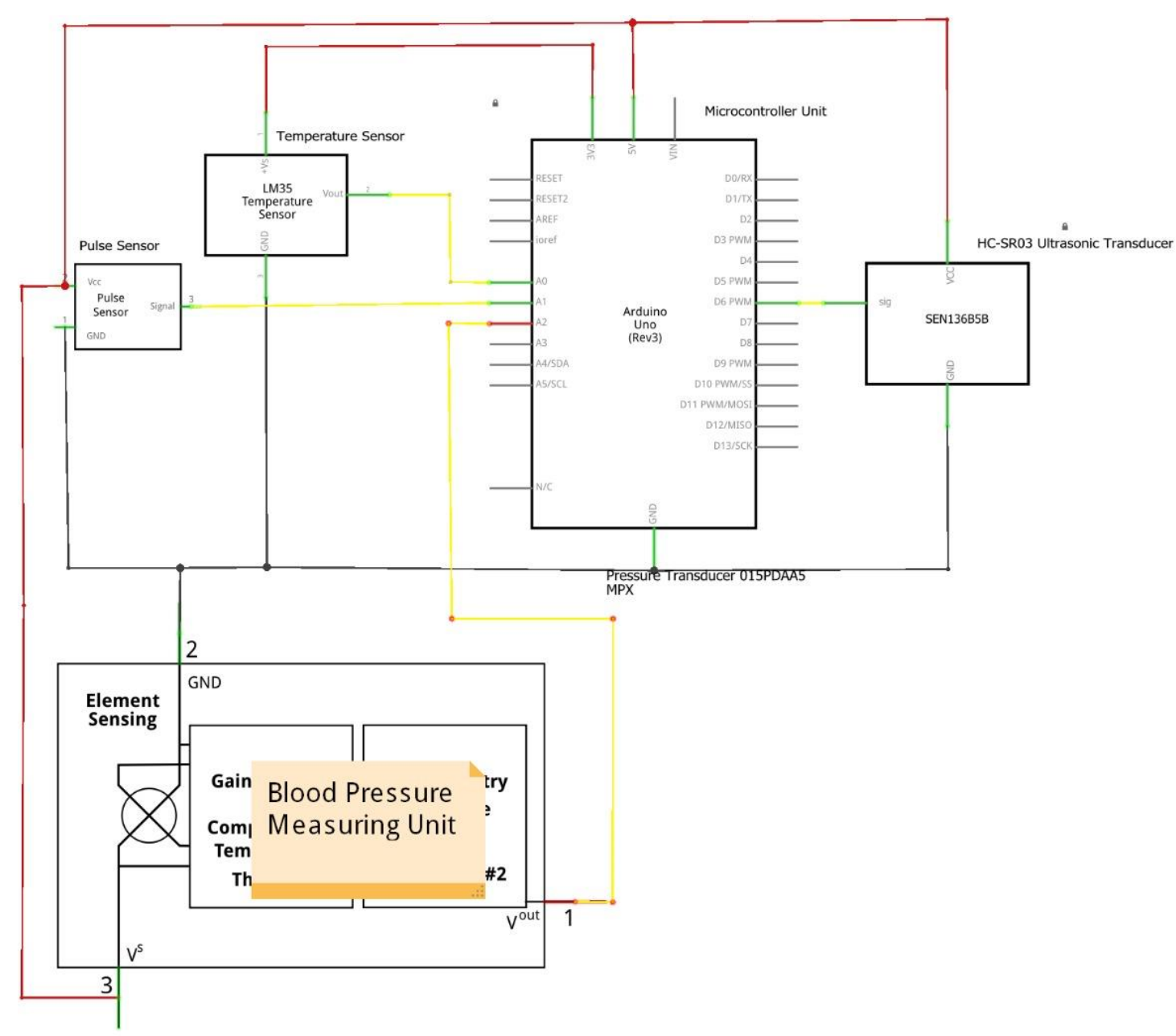

fritzing

Fig 2: Circuit Diagram of the proposed system

$66.46 \%$ percentage of the entire Indian population resides in rural areas. Unlike urban centres that have access to better doctors and quality clinics, the rural population is still grappling to find basic medical facilities. This machine will be an efficient solution to this beneficiary group. Although, it may take a while for them to get used to the system, on a longer run, they will no longer have to travel long distances to satisfy their health care needs. This can be for sure a great advancement in rural medicine especially for a country like India.

The kiosks are computer terminals that can guide the patients to access the right medicine and treatment at the time of need. Patients can also get notifications regarding medical camps, mobile medical help, important dates for vaccination, child care, insurance policies etc. It serves the patients to fulfil their basic needs such as measurement of height, weight, BMI, blood pressure and heart beat. Furthermore, live consultation facilities with specialized doctors through video and voice chats can improve the living standards of the rural society. The prescribed medicines can be instantly vended out from the kiosk. The machine completely eliminates the need for man power which in turn increases its working efficiency and effectiveness. Figure 3 shows the front end view of the proposed system. 


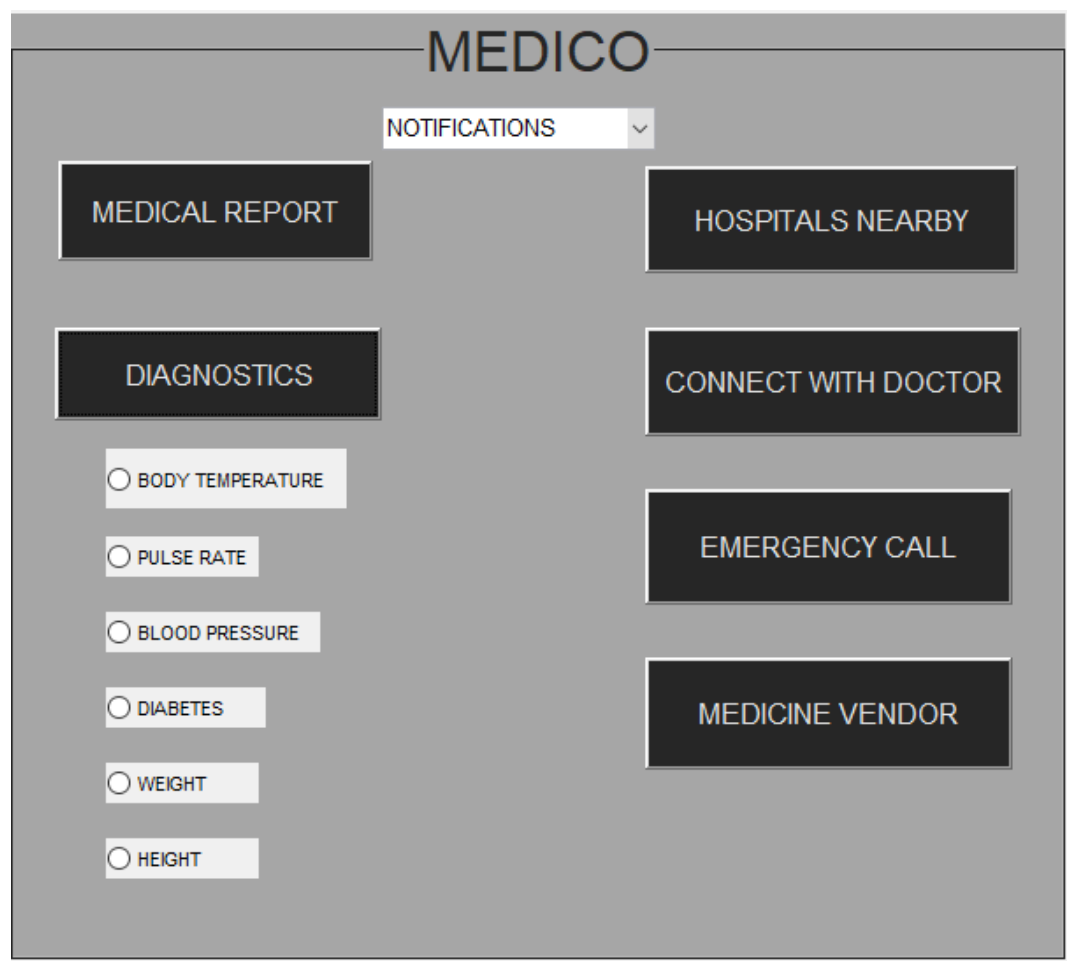

Fig 3: Front End view of the proposed system

\section{CONCLUSION}

The above proposed project will be a great innovation in the field of medicine in rural areas. The kiosk machine can be placed at any accessible point within the village so that the villagers will no longer have to travel long distances for medical aid. Since all updated information and medical facilities are readily available, this can definitely put an end to crisis faced, especially during emergency situations. This can also avoid fraud malpractices in the name of health care to a great extent. Ultimately, the culminated result can offer a new panorama in the field of rural medicine, proffering self-empowerment to the Indian villages.

\section{References}

[1] Data available online in https://en.wikipedia.org/wiki/Palissery [Accessed on 23rd Janyary 2019]

[2] Data available online in https://en.wikipedia.org/wiki/Karukutty_[Accessed on 23rd Janyary 2019]

[3] A. Al-Fuqaha, M. Guizani, M. Mohammadi, M. Aledhari and M. Ayyash, "Internet of Things: A Survey on Enabling Technologies, Protocols, and Applications," in IEEE Communications Surveys \& Tutorials, vol. 17, no. 4, pp. 2347-2376, Fourthquarter 2015.

[4] L. D. Xu, W. He and S. Li, "Internet of Things in Industries: A Survey," in IEEE Transactions on Industrial Informatics, vol. 10, no. 4, pp. 2233-2243, Nov. 2014. 
[5] Vinoj, P. G., Jacob, S., \& Menon, V. G. (2018, September). Hybrid brainactuated muscle interface for the physically disabled. In Basic \& Clinical Pharmacology \& Toxicology (Vol. 123, Pp. 8-9). 111 River St, Hoboken 07030-5774, USA: Wiley.

[6] Rajesh, S., Paul, V., Menon, V. G., \& Khosravi, M. R. (2019). A Secure and Efficient Lightweight Symmetric Encryption Scheme for Transfer of Text Files between Embedded IoT Devices. Symmetry, 11(2), 293.

[7] Alzubi, J., Jacob, S., Menon, V. G., Joseph, S., \& Vinoj, P. G. (2018, November). A TopUp Design for PAL to VGA Conversion in Real Time Video Processing System. In 2018 International Symposium on Advanced Electrical and Communication Technologies (ISAECT) (pp. 1-5). IEEE.

[8] Varun G Menon, "Light Weight Secure Encryption Scheme for Internet of Things Network", Encyclopedia, 2019.

[9] L. Catarinucci et al., "An IoT-Aware Architecture for Smart Healthcare Systems," in IEEE Internet of Things Journal, vol. 2, no. 6, pp. 515-526, Dec. 2015

[10] S. Jacob, "A Review of Technology Advances for Assisting Paralyzed People [Leading Edge]," in IEEE Technology and Society Magazine, vol. 36, no. 2, pp. 36-37, June 2017.

[11] A. Dohr, R. Modre-Opsrian, M. Drobics, D. Hayn and G. Schreier, "The Internet of Things for Ambient Assisted Living," 2010 Seventh International Conference on Information Technology: New Generations, Las Vegas, NV, 2010, pp. 804-809.

[12] Philip, V., Suman, V. K., Menon, V. G., \& Dhanya, K. A. (2017). A Review on latest Internet of Things based Healthcare Applications. International Journal of Computer Science and Information Security, 15(1), 248.

[13] Deshkar, S., Thanseeh, R. A., \& Menon, V. G. (2017). A Review on IoT based m-Health Systems for Diabetes. International Journal of Computer Science and Telecommunications, 8(1), 13-18

[14] F. Bonomi, R. Milito, J. Zhu and S. Addepalli, "Fog computing and its role in the internet of things," Proceedings of the first edition of the MCC workshop on Mobile cloud computing, Helsinki, Finland, 2012, pp. 13-16.

[15] Menon, Varun G., and Joe Prathap. "Vehicular Fog Computing: Challenges Applications and Future Directions." International Journal of Vehicular Telematics and Infotainment Systems (IJVTIS) 1.2 (2017): 15-23.

[16] Menon V G, Moving From Vehicular Cloud Computing to Vehicular Fog Computing: Issues and Challenges International Journal on Computer Science and Engineering, vol. 9, no. 2, pp. 14-18, 2017.

[17] S. Chakraborty, S. Bhowmick, P. Talaga and D. P. Agrawal, "Fog Networks in Healthcare Application," 2016 IEEE 13th International Conference on Mobile Ad Hoc and Sensor Systems (MASS), Brasilia, 2016, pp. 386-387.

[18] M. Al-khafajiy, L. Webster, T. Baker and A. Waraich, "Towards fog driven IoT healthcare: challenges and framework of fog computing in healthcare," Proceedings of the 2nd International Conference on Future Networks and Distributed Systems, Amman, Jordan, 2018, pp. 1-7. 